\title{
Joint Characteristic Function of Stock Log-Price and Squared Volatility in the Bates Model and Its Asset Pricing Applications
}

\author{
Oleksandr Zhylyevskyy \\ Department of Economics, Iowa State University, Ames, USA \\ Email: oz9a@iastate.edu
}

Received July 17, 2012; revised August 17, 2012; accepted September 17, 2012

\begin{abstract}
The model of Bates specifies a rich, flexible structure of stock dynamics suitable for applications in finance and economics, including valuation of derivative securities. This paper analytically derives a closed-form expression for the joint conditional characteristic function of a stock's log-price and squared volatility under the model dynamics. The use of the function, based on inverting it, is illustrated on examples of pricing European-, Bermudan-, and American-style options. The discussed approach for European-style derivatives improves on the option formula of Bates. The suggested approach for American-style derivatives, based on a compound-option technique, offers an alternative solution to existing finite-difference methods
\end{abstract}

Keywords: Bates Model; Stochastic Volatility; Jump-Diffusion; Characteristic Function; Option Pricing

\section{Introduction}

Stochastic volatility and jump-diffusion are standard tools of modeling asset price dynamics in finance research (see Ait-Sahalia and Jacod, 2011 [1]). Popularity of stochastic volatility models, such as the continuous-time model of Heston (1993) [2], is partly due to their ability to account for several aspects of stock price data that are not captured by analytically simpler geometric Brownian motion dynamics. For example, these models can help to account for an empirically relevant "leverage effect," which refers to an increase in the volatility of a stock when its price declines, and a decrease in the volatility when the price rises. They also can help to partly correct for deficiencies of the famous Black and Scholes (1973) [3] option pricing formula (e.g., the implied volatility "smile"). The model of Bates (1996) [4] extends the Heston model by incorporating jumps in stock dynamics. Allowing for jumps enables a more realistic representation of stock price time-series, which may feature discontinuities (for a discussion on jumps in asset data, see Ait-Sahalia and Jacod, 2009 [5]).

In this paper, I analytically derive and provide examples for the use of a closed-form expression for the joint conditional characteristic function of a stock's log-price and squared volatility under the dynamics of the Bates model. The model offers a rich distributional structure of stock returns. For instance, a skewed distribution can arise due to a correlation between shocks to the stock price and shocks to the volatility or due to nonzero average jumps. Excess kurtosis can arise from variable volatility or from a jump component. Also, the model can help to distinguish between two alternative explanations for skewness and excess kurtosis: stochastic volatility implies a positive relationship between the length of the holding period and the magnitude of skewness and kurtosis, whereas jumps imply a negative relationship (Bates, 1996 [4], pp. 72-73). The flexibility of the model makes it particularly attractive for the task of valuation of derivative securities. As such, it is useful in applied research and practice.

Under jump-diffusion dynamics with stochastic volatility, the values of derivative securities such as European-style options are typically impossible to express in simple form. Instead, they may be computed numerically by applying the transform methods of Duffie et al. (2000) [6] and Bakshi and Madan (2000) [7], which require inverting a conditional characteristic function of an underlying state-price vector. Bates (1996) [4] solved for the marginal conditional characteristic function of the logprice and derived a formula for the value of a European-style call option that involves two separate inversions. In contrast, the problem of finding the joint conditional characteristic function of the log-price and squared volatility was not posed, and to the best of my knowledge, a solution for this function is not available in 
existing finance studies. This paper aims to fill in the gap by deriving a closed-form expression for the function, which is an analytically challenging task. In addition, I provide two practically relevant examples illustrating the use of the function. The first example revisits the problem of the valuation of European-style options. I show that the marginal characteristic function is a special case of the joint characteristic function and then apply results from prior research to obtain formulas for European-style put and call options that require a single inversion; this approach is more efficient than the solution suggested by Bates involving two inversions. The second example addresses the problem of valuation of Bermudan- and American-style options by proposing an extension of the Geske-Johnson compound-option technique (Geske and Johnson, 1984 [8]). In this case, knowledge of the joint (rather than marginal) characteristic function is indispensable. The proposed approach provides an alternative to pricing American-style options using finite-difference methods (e.g., Chiarella et al., 2008 [9]), which can pose practical challenges when dealing with stochastic volatility (for a review, see Zhylyevskyy, 2010 [10]). The empirical relevance of the example is due to a large number of single name equity and commodity futures options traded on organized exchanges being Americanstyle.

The remainder of the paper is organized as follows. Section 2 sets up the Bates model and outlines the assumptions and notation. Section 3 derives a stochastic differential equation for the stock's log-price. Section 4 shows that the joint conditional characteristic function is a martingale and uses this result to derive a partial differential-integral equation for the function. Section 5 solves this equation analytically to obtain a closed-form expression for the function. Section 6 provides examples for the use of the function when pricing derivative securities. Section 7 concludes.

\section{The Bates Model}

I first outline the assumptions and introduce the notation. The financial market is assumed to admit no arbitrage opportunities. Thus, there is an equivalent martingale probability measure (see Harrison and Kreps, 1979 [11]), denoted here as $P^{1}$. Random variables and stochastic processes are defined on a probability space with $P$ as the probability measure. An expected value taken with respect to $P$ is denoted by $E[\cdot]$. To rigorously analyze stochastic processes, I work with a filtered probability space $\left(\Omega, \mathcal{F},\left\{\mathcal{F}_{t}\right\}_{t \geq 0}, P\right)$, where $\Omega$ is the set of outcomes, $t$ indexes time, $\left\{\mathcal{F}_{t}\right\}_{t>0}$ is a filtration (i.e., a non-decreasing sequence of $\sigma$-fields), and $\sigma$-field $\mathcal{F} \equiv \bigcup_{t} \mathcal{F}_{t}$. Stochastic processes are assumed to be

\footnotetext{
${ }^{1} P$ need not be unique, as the financial market may be incomplete.
}

adapted to $\left\{\mathcal{F}_{t}\right\}_{t \geq 0}$.

One of the assets traded in the financial market is a riskless bond fund with a share worth $M_{t}=M_{0} e^{r t}$ on date $t$, where $M_{0}>0$ is an initial value and $r \geq 0$ is a risk-free interest rate, which is assumed to be constant over time. In contexts involving asset pricing (e.g., valuation of derivative securities), such riskless fund is often used as a numéraire asset, with prices of other assets being discounted by $M_{t}$. Also, $P$ is often referred to as the "risk-neutral" probability measure.

I focus on a stock process $\left\{S_{t}\right\}_{t \geq 0}$, where $S_{t}$ denotes the price of the stock on date $t$. The stock is allowed to pay dividends continuously at a rate $\delta \geq 0$, which is assumed to be constant over time ( $\delta=0$ in the case of no dividend). Since the stock process in the Bates model incorporates a jump component, which results in discontinuities in the stock price, it is helpful to introduce the notion of a "left limit" of a stochastic process. In particular, the left limit of $\left\{S_{t}\right\}_{t \geq 0}$ on date $t$ is defined as $S_{t-}=\lim _{n \rightarrow \infty} S_{t-1 / n}$, where $n$ is a positive integer. If there is a jump on date $t$, then $S_{t-} \neq S_{t}$.

In the Bates model, the dynamics of $S_{t}$ under $P$ are described by a system of two stochastic differential equations:

$$
\begin{gathered}
d S_{t} / S_{t-}=(r-\delta-\theta \mu) d t+\sqrt{v_{t}} d W_{1 t}+U d N_{t}, \\
d v_{t}=\left(\alpha-\beta v_{t}\right) d t+\gamma \sqrt{v_{t}} d W_{2 t} .
\end{gathered}
$$

Equation (1) shows that the instantaneous net return on the stock, $d S_{t} / S_{t-}$, is a sum of three distinct components: 1) a deterministic drift term $(r-\delta-\theta \mu) d t ; 2)$ a stochastic diffusion term $\sqrt{v_{t}} d W_{1 t}$, and (3) a stochastic jump term $U d N_{t}$. A process $\left\{W_{1 t}\right\}_{t \geq 0}$ underlying the stochastic diffusion term is a standard Brownian motion. A process $\left\{N_{t}\right\}_{t \geq 0}$ underlying the stochastic jump term is a Poisson process with intensity $\theta \geq 0$, so that $E N_{t}=\theta t$. The processes $\left\{W_{1 t}\right\}_{t \geq 0}$ and $\left\{N_{t}\right\}_{t \geq 0}$ are independent of each other. A value of $N_{t}>0$ indicates that the stock price has undergone $N_{t}$ jumps as of date $t$. The magnitudes of such jumps are governed by independent and identically distributed (i.i.d.) random variables $U_{1}, U_{2}, \cdots$ such that

$$
\ln (1+U) \sim N\left[\ln (1+\mu)-\sigma^{2} / 2, \sigma^{2}\right],
$$

where $U$ is a generic random variable having the same distribution as $U_{1}, U_{2}, \cdots$, and $\mu>-1$ and $\sigma^{2} \geq 0$ are the distribution parameters. In Equation (1), $U$ is the random percentage jump of the stock price given a jump occurring at $t$ (i.e., given $d N_{t}=1$ ). The Bates model reduces to the Heston stochastic volatility model (Heston, 1993 [2]) if (1) $\theta=0$, or (2) $\mu=0$ and $\sigma^{2}=0$, since these cases effectively eliminate jumps from the stock dynamics.

Equation (2) describes a mean-reverting square root 
process for the squared volatility ${ }^{2}$. This equation is borrowed directly from the Heston model. A process $\left\{W_{2 t}\right\}_{t \geq 0}$ is a standard Brownian motion possibly correlated with $\left\{W_{1 t}\right\}_{t \geq 0}$, so that $d\left\langle W_{1}, W_{2}\right\rangle_{t}=\rho d t$, with $|\rho| \leq 1$. The process $\left\{W_{2 t}\right\}_{t \geq 0}$, process $\left\{N_{t}\right\}_{t \geq 0}$, and the random variables $U_{1}, U_{2}, \ldots$ are mutually independent. Constants $\alpha \geq 0, \beta \geq 0$, and $\gamma \geq 0$ are parameters. In order for $v_{t}$ to be almost surely (a.s.) positive so that $S_{t}$ and $v_{t}$ are real-valued a.s., $\alpha$ and $\gamma$ are assumed to satisfy a restriction $\gamma^{2} \leq 2 \alpha$ (see Chernov and Ghysels, 2000 [12]).

\section{Dynamics of Log-Price}

Let $S_{t}$ denote the stock's log-price, $s_{t}=\ln S_{t}$. The dynamics of $s_{t}$ under $P$ are derived using a generalized Itô formula for semimartingales, which allows me to properly account for possible discontinuities in the time path of the stock price. See Theorem 32 of Protter (1990 [13], p. 71) for details on the formula. Before applying the Itô formula, observe that Equation (1) implies that $S_{t}$ and $S_{t-}$ are, in general, not equal to each other because of the presence of the jump term; more specifically, $S_{t}-S_{t-}=S_{t-} U d N_{t}$. Thus, $S_{t} / S_{t-}=1+U d N_{t}$, and therefore,

$$
\ln S_{t}-\ln S_{t-}=\ln \left(1+U d N_{t}\right) .
$$

Also, note that by the properties of the Poisson process, $d N_{t}$ is effectively either 0 or 1 . Therefore,

$$
\ln S_{t}-\ln S_{t-}=\ln \left(1+U d N_{t}\right)=\ln (1+U) \cdot d N_{t} .
$$

Hence, the generalized Itô formula applied to the function $\ln \left(S_{t}\right)$ indicates that the dynamics of $S_{t}$ under $P$ are described by a stochastic differential equation

$$
\begin{aligned}
d s_{t}= & \frac{1}{S_{t-}} d S_{t}-\frac{1}{2} \frac{1}{\left(S_{t-}\right)^{2}} v_{t}\left(S_{t-}\right)^{2} d t \\
& +\ln S_{t}-\ln S_{t-}-\left(S_{t}-S_{t-}\right) / S_{t-},
\end{aligned}
$$

which is straightforward to simplify as:

$$
d s_{t}=\left(r-\delta-\theta \mu-v_{t} / 2\right) d t+\sqrt{v_{t}} d W_{1 t}+\ln (1+U) d N_{t} \text {. }
$$

\section{Martingale Property and Dynamics of Joint Characteristic Function}

My main interest lies in deriving a closed-form expression for the joint characteristic function of some future, date- $T$ log-price and squared volatility given their present, date- $t$ values, where $t<T$. Consider an arbitrary date $h$ such that $t<h \leq T$, and note that since $t<h, \mathcal{F}_{t} \subset \mathcal{F}_{h}$. Equations (2) and (4), the

\footnotetext{
${ }^{2}$ In applications, the unobserved value of $v_{t}$ is often treated as an additional parameter to estimate.
}

properties of the Poisson process and standard Brownian motion, and the assumption of i.i.d. random variables $U_{1}, U_{2}, \cdots$ imply that the information contained in the $\sigma$-field $\mathcal{F}_{h}$ relevant for conditioning the joint distribution of $s_{T}$ and $v_{T}$ on $\mathcal{F}_{h}$ comprises the values of $s_{h}$ and $v_{h}$, and the time remaining at $h$ until $T$, $T-h \geq 0$. Thus, let $\Psi\left(\zeta_{1}, \zeta_{2} ; s_{h}, v_{h}, T-h\right)$ denote the joint conditional characteristic function of $\left(s_{T}, v_{T}\right)$ given $\left(s_{h}, v_{h}\right)$, evaluated at real arguments $\zeta_{1}$ and $\zeta_{2}$. By definition of the characteristic function,

$$
\Psi\left(\zeta_{1}, \zeta_{2} ; s_{h}, v_{h}, T-h\right)=E\left[e^{i\left(\zeta_{1} s_{T}+\zeta_{2} v_{T}\right)} \mid \mathcal{F}_{h}\right] .
$$

Likewise,

$$
\Psi\left(\zeta_{1}, \zeta_{2} ; s_{h}, v_{h}, T-t\right)=E\left[e^{i\left(\zeta_{1} s_{T}+\zeta_{2} v_{T}\right)} \mid \mathcal{F}_{t}\right] .
$$

Since $\mathcal{F}_{t} \subset \mathcal{F}_{h}$, the law of iterated expectations implies that

$$
\begin{aligned}
\Psi\left(\zeta_{1}, \zeta_{2} ; s_{t}, v_{t}, T-t\right) & =E\left[e^{i\left(\zeta_{1} s_{T}+\zeta_{2} v_{T}\right)} \mid \mathcal{F}_{t}\right] \\
& =E\left[E\left[e^{i\left(\zeta_{1} s_{T}+\zeta_{2} v_{T}\right)} \mid \mathcal{F}_{h}\right] \mid \mathcal{F}_{t}\right] \\
& =E\left[\Psi\left(\zeta_{1}, \zeta_{2} ; s_{h}, v_{h}, T-h\right) \mid \mathcal{F}_{t}\right] \text { a.s., }
\end{aligned}
$$

which shows that

$$
\left\{\Psi\left(\zeta_{1}, \zeta_{2} ; s_{t}, v_{t}, T-t\right)\right\}_{t: 0 \leq t \leq T}
$$

is a martingale. The martingale property of $\Psi(\cdot)$ implies that

$$
E\left[d \Psi\left(\zeta_{1}, \zeta_{2} ; s_{t}, v_{t}, T-t\right) \mid \mathcal{F}_{t-}\right]=0 \text { a.s. }
$$

In what follows, it is helpful to denote by $\tau$ the duration of the time interval between $t$ and $T, \tau=T-t$. Also, observe that Equation (2) implies that $v_{t}$ has a continuous time path; therefore, $v_{t-}=v_{t}$. In comparison, Equation (4) implies that $s_{t}$ may have discontinuities in its path, with $s_{t}=s_{t-}+\ln (1+U) d N_{t}$.

The goal is to find a solution for $\Psi(\cdot)$ as a function with continuous second order partial derivatives. Applying the generalized Itô formula,

$$
\begin{aligned}
d \Psi\left(\zeta_{1}, \zeta_{2} ; s_{t}, v_{t}, \tau\right) & =-\Psi_{\tau}\left(\zeta_{1}, \zeta_{2} ; s_{t-}, v_{t}, \tau\right) d t \\
& +\Psi_{s}\left(\zeta_{1}, \zeta_{2} ; s_{t-}, v_{t}, \tau\right) \\
& \times\left[\left(r-\delta-\theta \mu-v_{t} / 2\right) d t+\sqrt{v_{t}} d W_{1 t}\right] \\
& +\Psi_{v}\left(\zeta_{1}, \zeta_{2} ; s_{t-}, v_{t}, \tau\right) \\
& \times\left[\left(\alpha-\beta v_{t}\right) d t+\gamma \sqrt{v_{t}} d W_{2 t}\right] \\
& +0.5 \Psi_{s s}\left(\zeta_{1}, \zeta_{2} ; s_{t-}, v_{t}, \tau\right) \cdot v_{t} d t \\
& +0.5 \Psi_{v v}\left(\zeta_{1}, \zeta_{2} ; s_{t-}, v_{t}, \tau\right) \cdot \gamma^{2} v_{t} d t \\
& +\Psi_{s v}\left(\zeta_{1}, \zeta_{2} ; s_{t-}, v_{t}, \tau\right) \cdot \rho \gamma v_{t} d t \\
& +\Psi\left(\zeta_{1}, \zeta_{2} ; s_{t-}+\ln (1+U) d N_{t}, v_{t}, \tau\right) \\
& -\Psi\left(\zeta_{1}, \zeta_{2} ; s_{t-}, v_{t}, \tau\right)
\end{aligned}
$$


where symbolic terms of the form $\Psi_{x}$ and $\Psi_{x y}$ denote partial derivatives $\partial \Psi / \partial x$ and $\partial^{2} \Psi / \partial x \partial y$, respectively.

By the properties of the Poisson process and mutual independence of $U$ and $d N_{t}$,

$$
\begin{aligned}
E[\Psi & \left(\zeta_{1}, \zeta_{2} ; s_{t-}+\ln (1+U) d N_{t}, v_{t}, \tau\right) \\
& \left.-\Psi\left(\zeta_{1}, \zeta_{2} ; s_{t-}, v_{t}, \tau\right) \mid \mathcal{F}_{t-}\right] \\
=\theta\{ & E\left[\Psi\left(\zeta_{1}, \zeta_{2} ; s_{t-}+\ln (1+U), v_{t}, \tau\right) \mid \mathcal{F}_{t-}\right] \\
& \left.-\Psi\left(\zeta_{1}, \zeta_{2} ; s_{t-}, v_{t}, \tau\right)\right\} d t .
\end{aligned}
$$

Then, applying the relationship $E\left[d \Psi(\cdot) \mid \mathcal{F}_{t-}\right]=0$ and the properties of the standard Brownian motion, it is straightforward to show that the function $\Psi(\cdot)$ must satisfy the following partial differential-integral equation:

$$
\begin{aligned}
0= & -\Psi_{\tau}+\Psi_{s} \cdot\left(r-\delta-\theta \mu-v_{t} / 2\right)+\Psi_{v} \cdot\left(\alpha-\beta v_{t}\right) \\
+ & \Psi_{s s} \cdot v_{t} / 2+\Psi_{v v} \cdot \gamma^{2} v_{t} / 2+\Psi_{s v} \cdot \rho \gamma v_{t} \\
+ & \theta\left\{E\left[\Psi\left(\zeta_{1}, \zeta_{2} ; s_{t-}+\ln (1+U), v_{t}, \tau\right) \mid \mathcal{F}_{t-}\right]\right. \\
& \left.\quad-\Psi\left(\zeta_{1}, \zeta_{2} ; s_{t-}, v_{t}, \tau\right)\right\},
\end{aligned}
$$

where the arguments of the partial derivatives and the term $d t$ are omitted to shorten the notation. Note that in the special case of $\tau=0, \Psi(\cdot)=\exp \left[i\left(\zeta_{1} s_{T}+\zeta_{2} v_{T}\right)\right]$.

\section{Closed-Form Solution for Joint Characteristic Function}

Solving for the joint characteristic function $\Psi(\cdot)$ in closed form using Equation (5) presents a substantial analytical challenge. My approach to address this problem is to first propose a general form of a solution to the equation, and then analytically derive all of the solution components. Suppose that $\Psi(\cdot)$ is of the form:

$$
\begin{aligned}
\Psi\left(\zeta_{1}, \zeta_{2} ; s_{t}, v_{t}, \tau\right)=\exp [ & p\left(\tau ; \zeta_{1}, \zeta_{2}\right)+q\left(\tau ; \zeta_{1}, \zeta_{2}\right) v_{t} \\
& \left.+i \zeta_{1} s_{t}+\kappa\left(\zeta_{1}\right) \tau\right]
\end{aligned}
$$

where $p\left(\tau ; \zeta_{1}, \zeta_{2}\right)$ and $q\left(\tau ; \zeta_{1}, \zeta_{2}\right)$ are complexvalued functions of $\tau$ to be solved for analytically, and $\kappa\left(\zeta_{1}\right)$ is complex-valued and constant with respect to $s_{t}, v_{t}$, and $\tau$. The expression for $\kappa$ is provided shortly.

Differentiating the expression for $\Psi(\cdot)$ in Equation (6):

$$
\begin{aligned}
& \Psi_{\tau}\left(\zeta_{1}, \zeta_{2} ; s_{t-}, v_{t}, \tau\right) \\
&= \Psi\left(\zeta_{1}, \zeta_{2} ; s_{t-}, v_{t}, \tau\right) \\
& \times\left[p_{\tau}\left(\tau ; \zeta_{1}, \zeta_{2}\right)+q_{\tau}\left(\tau ; \zeta_{1}, \zeta_{2}\right) v_{t}+\kappa\left(\zeta_{1}\right)\right], \\
& \Psi_{s}\left(\zeta_{1}, \zeta_{2} ; s_{t-}, v_{t}, \tau\right)=\Psi\left(\zeta_{1}, \zeta_{2} ; s_{t-}, v_{t}, \tau\right) \cdot i \zeta_{1}, \\
& \Psi_{v}\left(\zeta_{1}, \zeta_{2} ; s_{t-}, v_{t}, \tau\right)=\Psi\left(\zeta_{1}, \zeta_{2} ; s_{t-}, v_{t}, \tau\right) \cdot q\left(\tau ; \zeta_{1}, \zeta_{2}\right),
\end{aligned}
$$

$$
\begin{aligned}
& \Psi_{s s}\left(\zeta_{1}, \zeta_{2} ; s_{t-}, v_{t}, \tau\right)=\Psi\left(\zeta_{1}, \zeta_{2} ; s_{t-}, v_{t}, \tau\right) \cdot\left[i \zeta_{1}\right]^{2}, \\
& \Psi_{v v}\left(\zeta_{1}, \zeta_{2} ; s_{t-}, v_{t}, \tau\right)=\Psi\left(\zeta_{1}, \zeta_{2} ; s_{t-}, v_{t}, \tau\right) \cdot\left[q\left(\tau ; \zeta_{1}, \zeta_{2}\right)\right]^{2},
\end{aligned}
$$

and

$$
\begin{aligned}
& \Psi_{s v}\left(\zeta_{1}, \zeta_{2} ; s_{t-}, v_{t}, \tau\right) \\
& =\Psi\left(\zeta_{1}, \zeta_{2} ; s_{t-}, v_{t}, \tau\right) \times i \zeta_{1} \cdot q\left(\tau ; \zeta_{1}, \zeta_{2}\right),
\end{aligned}
$$

where $\quad p_{\tau}(\cdot)=d p(\cdot) / d \tau$ and $q_{\tau}(\cdot)=d q(\cdot) / d \tau$.

Next, recall from Equation (3) that $\ln (1+U)$ is a normal random variable. By assumption, it is independent of the information contained in $\mathcal{F}_{t-}$. Thus, using Equation (6),

$$
\begin{aligned}
& E\left[\Psi\left(\zeta_{1}, \zeta_{2} ; s_{t-}+\ln (1+U), v_{t}, \tau\right) \mid \mathcal{F}_{t-}\right] \\
& -\Psi\left(\zeta_{1}, \zeta_{2} ; s_{t-}, v_{t}, \tau\right) \\
= & E\left[\Psi\left(\zeta_{1}, \zeta_{2} ; s_{t-}, v_{t}, \tau\right) \cdot \exp \left[i \zeta_{1} \ln (1+U)\right] \mid \mathcal{F}_{t-}\right] \\
- & \Psi\left(\zeta_{1}, \zeta_{2} ; s_{t-}, v_{t}, \tau\right) \\
= & \Psi\left(\zeta_{1}, \zeta_{2} ; s_{t-}, v_{t}, \tau\right) \cdot\left\{E\left[\exp \left[i \zeta_{1} \ln (1+U)\right]\right]-1\right\} \\
= & \Psi\left(\zeta_{1}, \zeta_{2} ; s_{t-}, v_{t}, \tau\right) \\
& \times\left\{\exp \left[i \zeta_{1} \ln (1+\mu)-\left(i+\zeta_{1}\right) \zeta_{1} \sigma^{2} / 2\right]-1\right\},
\end{aligned}
$$

where the last equality follows from the properties of the characteristic function of a normal random variable (see Chung, 2001 [14], p. 156). Given this result, it is convenient to express $\kappa$ as follows:

$$
\kappa\left(\zeta_{1}\right)=\theta\left\{\exp \left[i \zeta_{1} \ln (1+\mu)-\left(i+\zeta_{1}\right) \zeta_{1} \sigma^{2} / 2\right]-1\right\} .
$$

Then, the integral term in Equation (5) is

$$
\begin{aligned}
\theta\{ & {\left[\Psi\left(\zeta_{1}, \zeta_{2} ; s_{t-}+\ln (1+U), v_{t}, \tau\right) \mid \mathcal{F}_{t-}\right] } \\
- & \left.\Psi\left(\zeta_{1}, \zeta_{2} ; s_{t-}, v_{t}, \tau\right)\right\} \\
= & \Psi\left(\zeta_{1}, \zeta_{2} ; s_{t-}, v_{t}, \tau\right) \cdot \kappa\left(\zeta_{1}\right) .
\end{aligned}
$$

By plugging in the obtained expressions into Equation (5) and simplifying it (e.g., note that $\kappa$ is differenced out), I get

$$
\begin{aligned}
0= & {\left[-p_{\tau}\left(\tau ; \zeta_{1}, \zeta_{2}\right)+i \zeta_{1}(r-\delta-\theta \mu)+\alpha q\left(\tau ; \zeta_{1}, \zeta_{2}\right)\right]+v_{t} } \\
& \times\left[-q_{\tau}\left(\tau ; \zeta_{1}, \zeta_{2}\right)-i \zeta_{1} / 2-\beta q\left(\tau ; \zeta_{1}, \zeta_{2}\right)-\zeta_{1}^{2} / 2\right. \\
& \left.+\gamma^{2}\left[q\left(\tau ; \zeta_{1}, \zeta_{2}\right)\right]^{2} / 2+i \zeta_{1} \rho \gamma q\left(\tau ; \zeta_{1}, \zeta_{2}\right)\right] .
\end{aligned}
$$

Since this equation must hold irrespective of a particular value of $v_{t}$, the functions $p(\cdot)$ and $q(\cdot)$ need to solve the following system of two differential equations:

$$
p_{\tau}\left(\tau ; \zeta_{1}, \zeta_{2}\right)=i \zeta_{1}(r-\delta-\theta \mu)+\alpha q\left(\tau ; \zeta_{1}, \zeta_{2}\right),
$$




$$
\begin{aligned}
q_{\tau}\left(\tau ; \zeta_{1}, \zeta_{2}\right)= & -\zeta_{1}^{2} / 2-i \zeta_{1} / 2+\left[i \zeta_{1} \rho \gamma-\beta\right] q\left(\tau ; \zeta_{1}, \zeta_{2}\right) \\
& +\gamma^{2}\left[q\left(\tau ; \zeta_{1}, \zeta_{2}\right)\right]^{2} / 2
\end{aligned}
$$

Observe that the relationship

$$
\begin{aligned}
\Psi\left(\zeta_{1}, \zeta_{2} ; s_{T}, v_{T}, 0\right) \equiv \exp [ & \left.i\left(\zeta_{1} s_{T}+\zeta_{2} v_{T}\right)\right] \\
=\exp [ & p\left(0 ; \zeta_{1}, \zeta_{2}\right) \\
& \left.+q\left(0 ; \zeta_{1}, \zeta_{2}\right) v_{T}+i \zeta_{1} s_{T}\right]
\end{aligned}
$$

implies that the system has initial conditions $p\left(0 ; \zeta_{1}, \zeta_{2}\right)=0$ and $q\left(0 ; \zeta_{1}, \zeta_{2}\right)=i \zeta_{2}$.

The system is similar, although not identical, to a system of differential equations analyzed by Zhylyevskyy (2010) [10] in the case of the Heston model. By appropriately modifying the prior analysis, a closed-form solution for $p(\cdot)$ and $q(\cdot)$, in the case of the Bates model studied here, can be split into three cases. In Case (1) below, parameter $\gamma \neq 0$, which implies that the squared volatility process is stochastic. In comparison, Case (2) and Case (3) provide solutions for $p(\cdot)$ and $q(\cdot)$ when $\gamma=0$, that is, under the special circumstances of non-stochastic stock volatility ${ }^{3}$.

Case (1). Suppose that $\gamma \neq 0$. Let $A\left(\zeta_{1}\right)$ and $B\left(\zeta_{1}, \zeta_{2}\right)$ be complex-valued and constant with respect to $\tau$, and defined as follows:

$$
\begin{gathered}
A\left(\zeta_{1}\right)=\gamma^{2}\left(1-\rho^{2}\right) \zeta_{1}^{2}+\left(\gamma^{2}-2 \rho \gamma \beta\right) i \zeta_{1}+\beta^{2}, \\
B\left(\zeta_{1}, \zeta_{2}\right)=-\frac{\rho \gamma i \zeta_{1}-\beta-\sqrt{A\left(\zeta_{1}\right)}+\gamma^{2} i \zeta_{2}}{\rho \gamma i \zeta_{1}-\beta+\sqrt{A\left(\zeta_{1}\right)}+\gamma^{2} i \zeta_{2}} .
\end{gathered}
$$

Then,

$$
\begin{aligned}
p\left(\tau ; \zeta_{1}, \zeta_{2}\right) & =\tau(r-\delta-\theta \mu-\alpha \rho / \gamma) i \zeta_{1} \\
& +\frac{\alpha}{\gamma^{2}}\left[\tau \beta+\tau \sqrt{A}+2 \ln \left(\frac{B+1}{B e^{\tau \sqrt{A}}+1}\right)\right], \\
q\left(\tau ; \zeta_{1}, \zeta_{2}\right)= & \frac{1}{\gamma^{2}}\left[\beta-\rho \gamma i \zeta_{1}-\sqrt{A} \frac{B e^{\tau \sqrt{A}}-1}{B e^{\tau \sqrt{A}}+1}\right],
\end{aligned}
$$

where $A \equiv A\left(\zeta_{1}\right)$ and $B \equiv B\left(\zeta_{1}, \zeta_{2}\right)$ as defined above.

Case (2). Suppose that $\gamma=0$ but $\beta \neq 0$. Then,

$$
\begin{aligned}
p\left(\tau ; \zeta_{1}, \zeta_{2}\right)= & \tau(r-\delta-\theta \mu) i \zeta_{1} \\
& -\frac{\alpha}{2 \beta}\left[2\left(e^{-\beta \tau}-1\right) i \zeta_{2}+\tau i \zeta_{1}+\tau \zeta_{1}^{2}\right] \\
& -\frac{\alpha\left(e^{-\beta \tau}-1\right)}{2 \beta^{2}}\left[\zeta_{1}^{2}+i \zeta_{1}\right],
\end{aligned}
$$

${ }^{3}$ Observe from Equation (2) that the value of $\gamma=0$ eliminates the diffusion component from the dynamics of $v_{t}$.

$$
q\left(\tau ; \zeta_{1}, \zeta_{2}\right)=\frac{1}{2 \beta}\left[e^{-\beta \tau}\left(\zeta_{1}^{2}+i \zeta_{1}+2 \beta i \zeta_{2}\right)-\zeta_{1}^{2}-i \zeta_{1}\right]
$$

Case (3). Suppose that $\gamma=0$ and $\beta=0$. Then,

$$
\begin{aligned}
p\left(\tau ; \zeta_{1}, \zeta_{2}\right) & =\tau(r-\delta-\theta \mu) i \zeta_{1} \\
& -\frac{\alpha \tau}{4}\left[\tau\left(\zeta_{1}^{2}+i \zeta_{1}\right)-4 i \zeta_{2}\right], \\
q\left(\tau ; \zeta_{1}, \zeta_{2}\right) & =-\frac{\tau}{2}\left[\zeta_{1}^{2}+i \zeta_{1}\right]+i \zeta_{2} .
\end{aligned}
$$

Together, the expression for $\Psi(\cdot)$ in Equation (6), the expression for $\kappa$ in Equation (7), and the solution for $p(\cdot)$ and $q(\cdot)$ given by Equations (8) and (9), respectively (alternatively, Equations (10) and (11) or Equations (12) and (13), respectively, depending on the particular values of $\gamma$ and $\beta$, as shown above), provide a closed-form, analytical expression for the joint characteristic function of $s_{T}$ and $v_{T}$, conditional on $s_{t}$ and $v_{t}$.

\section{Applications of Joint Characteristic Function}

The derived joint characteristic function may be employed in asset pricing applications. To illustrate its use, I provide two examples related to implementing the transform methods of Duffie et al. (2000) [6] and Bakshi and Madan (2000) [7] to price derivative securities under the dynamics of the Bates model. These methods require inverting a conditional characteristic function of an underlying state-price vector. Thus, knowledge of a closedform expression for the characteristic function, such as the one obtained in this paper, is essential for their implementation.

In the first example, I consider the problem of determining the values of European-style derivative securities. Let $P^{E}\left(X, S_{t}, v_{t}, \tau\right)$ denote the date- $t$ value of a European-style put option with a strike price $X$ and time to expiration $\tau$, given the (current) underlying stock's price $S_{t}$ and squared volatility $v_{t}$. This put option is allowed to be exercised on date $T$, and its date- $T$ value is $P^{E}\left(X, S_{T}, v_{T}, 0\right)=\max \left\{0, X-S_{T}\right\}$. Likewise, let $C^{E}\left(X, S_{t}, v_{t}, \tau\right)$ be the date- $t$ value of a corresponding European-style call option; its date- $T$ value is $C^{E}\left(X, S_{T}, v_{T}, 0\right)=\max \left\{0, S_{T}-X\right\}$. The dynamics of $S_{t}$ and $v_{t}$, under the equivalent martingale probability measure, are described by Equations (1) and (2). Thus, there are two state variables (comprising the state-price vector), namely, $S_{t}$ and $v_{t}$, or equivalently, (the logprice) $S_{t}$ and $v_{t}$.

The analysis of Zhylyevskyy (2012) [15] adapted to the case of the Bates model indicates that the valuation of the options $P^{E}(\cdot)$ and $C^{E}(\cdot)$ requires knowledge of 
the marginal conditional characteristic function of the date- $T \log$-price $S_{T}$ given $S_{t}$ and $v_{t}$. Let this function be denoted as $\varphi\left(\zeta ; s_{t}, v_{t}, \tau\right)$, where $\zeta$ is a real number. By definition,

$$
\varphi\left(\zeta ; s_{t}, v_{t}, \tau\right)=E\left[e^{i \zeta s_{T}} \mid \mathcal{F}_{t}\right]
$$

A closed-form expression for $\varphi\left(\zeta ; s_{t}, v_{t}, \tau\right)$ is easily obtained as a special case of the expression for $\Psi\left(\zeta_{1}, \zeta_{2} ; s_{t}, v_{t}, \tau\right)$, which was derived earlier. Namely,

$$
\begin{aligned}
\varphi\left(\zeta ; s_{t}, v_{t}, \tau\right) & =E\left[e^{i \zeta s_{T}} \mid \mathcal{F}_{t}\right] \\
& =E\left[e^{i\left(\zeta s_{T}+0 \cdot v_{T}\right)} \mid \mathcal{F}_{t}\right] \\
& =\Psi\left(\zeta, 0 ; s_{t}, v_{t}, \tau\right) .
\end{aligned}
$$

Then, applying Equation (10) of Zhylyevskyy (2012) [15], the value of $P^{E}(\cdot)$ is

$$
\begin{aligned}
& P^{E}\left(X, S_{t}, v_{t}, \tau\right) \\
= & \frac{1}{2} e^{-r \tau} X \times\left[1-\frac{2}{\pi} \int_{0}^{\infty} \operatorname{Re}\left[\frac{X^{-i \zeta}}{i \zeta+\zeta^{2}} \varphi\left(\zeta ; s_{t}, v_{t}, \tau\right)\right] \mathrm{d} \zeta\right],
\end{aligned}
$$

where $R e[\cdot]$ denotes the real part of a complex-valued number. In turn, $C^{E}(\cdot)$ can be calculated using a putcall parity relationship for European-style options (Merton, 1973 [16]):

$$
C^{E}\left(X, S_{t}, v_{t}, \tau\right)=P^{E}\left(X, S_{t}, v_{t}, \tau\right)+e^{-\delta \tau} S_{t}-e^{-r \tau} X .
$$

A practical implementation of these formulas for $P^{E}(\cdot)$ and $C^{E}(\cdot)$ would require numerical integration to calculate the term $\int \operatorname{Re}[\cdot] \mathrm{d} \zeta$, which is straightforward, using the Gauss-Kronrod quadrature method, for example (Press et al., 2001 [17]). Notably, these formulas provide a more efficient approach to price European-style derivative securities under the Bates model dynamics than the original solution of Bates. For instance, the formula for $C^{E}(\cdot)$ proposed here requires a single numerical integration, whereas the corresponding formula due to Bates (see Bates, 1996 [4], Equation (15) on p. 77) requires two separate integrations.

In the second example, I consider the problem of pricing Bermudan- and American-style options by building on the methodological approach developed by Zhylyevskyy (2010) [10]. The approach extends the Geske-Johnson compound-option technique (Geske and Johnson, 1984 [8]) to a case of non-Black-Scholes stock dynamics. In comparison to the first example in this section, which utilizes only a special case

$$
\varphi\left(\zeta ; s_{t}, v_{t}, \tau\right)=\Psi\left(\zeta, 0 ; s_{t}, v_{t}, \tau\right)
$$

of the joint characteristic function $\Psi(\cdot)$, this second example requires knowledge of the value of $\Psi\left(\zeta_{1}, \zeta_{2} ; s_{t}, v_{t}, \tau\right)$ for any combination of real numbers $\zeta_{1}$ and $\zeta_{2}$, including cases of $\zeta_{2} \neq 0$.

Let $f\left(s_{T}, v_{T} ; s_{t}, v_{t}, \tau\right)$ be the joint probability density function of $S_{T}$ and $v_{T}$, conditional on $s_{t}$ and $v_{t}$. The density function $f(\cdot)$ is an inverse Fourier transform of the characteristic function $\Psi(\cdot)$ (see Shephard, 1991 [18]; Chung, 2001 [14]):

$$
\begin{aligned}
& f\left(s_{T}, v_{T} ; s_{t}, v_{t}, \tau\right) \\
= & \frac{1}{(2 \pi)^{2}} \times \int_{-\infty}^{\infty} \int_{-\infty}^{\infty} e^{-i\left(\zeta_{1} s_{T}+\zeta_{2} v_{T}\right)} \Psi\left(\zeta_{1}, \zeta_{2} ; s_{t}, v_{t}, \tau\right) \mathrm{d} \zeta_{1} \mathrm{~d} \zeta_{2} .
\end{aligned}
$$

In practice, numerical values of $f(\cdot)$ can be efficiently computed using values of $\Psi(\cdot)$ by applying a fast Fourier transform algorithm with kernel smoothing (see Press et al., 2001 [17]; Zhylyevskyy, 2010 [10]).

American-style put and call options are similar to their European-style counterparts, except that the Americanstyle ones are allowed to be exercised at any time before expiration (the European-style ones may be exercised only on the expiration date). The possibility of an early exercise substantially complicates the problem of determining the value of an American-style derivative security; thus, closed-form solutions are generally not available (Epps, 2000 [19]). Bermudan-style options may be viewed as an intermediate case between their European- and American-style counterparts. A Bermudanstyle option is allowed to be exercised before expiration, but only on a selected number of predetermined dates. To clarify the idea, let

$$
\left\{D_{n}\left(s_{t}, v_{t}, T-t\right)\right\}_{n=1}^{\infty}
$$

be a sequence of Bermudan-style options, where $D_{n}(\cdot)$ is the value of an option that may be exercised on dates

$$
t_{j}=t+\frac{j(T-t)}{n}
$$

for $j=1, \cdots, n$. In the sequence, $D_{1}(\cdot)$ represents the value of a European-style option, which may be exercised only once, on the expiration date, with $n=1$ and $t_{1}=T \cdot D_{2}(\cdot)$ is the value of a Bermudan-style option that may be exercised on two dates, $t_{1}=(t+T) / 2$ (i.e., half-way to expiration) and $t_{2}=T . D_{3}(\cdot), D_{4}(\cdot), \cdots$ are defined similarly. The limit of the sequence, $D_{\infty}(\cdot)$, corresponds to the value of an American-style option, which features a continuum of possible exercise dates before expiration.

Let the exercise value of the Bermudan-style option $D_{n}(\cdot)$ on its first potential exercise date $t_{1}>t$ be denoted as

$$
\mathcal{X}\left(s_{t_{1}}, v_{t_{1}}, T-t_{1}\right) .
$$

For example,

$$
\mathcal{X}(\cdot)=\max \left\{0, X-e^{s_{t_{1}}}\right\}
$$


in the case of a put option and

$$
\mathcal{X}(\cdot)=\max \left\{0, e^{s_{t_{1}}}-X\right\}
$$

in the case of a call option. Bermudan-style derivative securities obey a recursive relationship:

$$
\begin{aligned}
& D_{n}\left(s_{t}, v_{t}, T-t\right) \\
= & e^{-r\left(t_{1}-t\right)} \\
& \times E\left[\max \left\{\mathcal{X}\left(s_{t_{1}}, v_{t_{1}}, T-t_{1}\right), D_{n-1}\left(s_{t_{1}}, v_{t_{1}}, T-t_{1}\right)\right\} \mid \mathcal{F}_{t}\right] \\
= & e^{-r\left(t_{1}-t\right)} \int_{-\infty 0}^{\infty} \int_{-\infty}^{\infty} \max \left\{\mathcal{X}\left(s, v, T-t_{1}\right), D_{n-1}\left(s, v, T-t_{1}\right)\right\} \\
& \times f\left(s, v ; s_{t}, v_{t}, t_{1}-t\right) d v d s,
\end{aligned}
$$

where $D_{0} \equiv 0$ and $f\left(s, v ; s_{t}, v_{t}, t_{1}-t\right)$ can be computed by inverting $\Psi\left(\zeta_{1}, \zeta_{2} ; s_{t}, v_{t}, t_{1}-t\right)$, as discussed earlier. In theory, the relationship provides a way to compute the price of any Bermudan-style derivative security, as well as to approximate the price of an Americanstyle one by choosing a sufficiently large value of $n$. In practice, it should be straightforward to compute $D_{1}\left(s_{t}, v_{t}, T-t\right)$ and $D_{2}\left(s_{t}, v_{t}, T-t\right)$, and if computationally feasible, $D_{3}\left(s_{t}, v_{t}, T-t\right)$, and then use these computed values to approximate the American-style option price $D_{\infty}\left(s_{t}, v_{t}, T-t\right)$ by applying a Richardson extrapolation (e.g., see Zhylyevskyy, 2010 [10]). This methodological approach is an alternative to pricing American-style derivative securities under the Bates model dynamics using a finite-difference-type scheme proposed by Chiarella et al. (2008) [9] $]^{4}$.

\section{Conclusion}

This paper contributes to the literature by solving in closed form for the joint conditional characteristic function of the log-price and squared volatility under the jump-diffusion dynamics of the Bates model. The model features a flexible distributional structure of asset returns. As such, it has a number of applications in finance and economics, including the problem of valuation of derivative securities. Obtaining a closed-form expression for the joint characteristic function is an analytically demanding task, which involves applying a generalized Itô formula for semimartingales and solving a system of differential equations, among other steps. The use of the derived function is illustrated on empirically relevant examples of pricing European-, Bermudan-, and American-style options. The proposed methodological approach is based on inverting the characteristic function, and may be em-

${ }^{4}$ Chiarella et al. propose a method of lines, in which a partial differential-integral equation is replaced with a system of simpler differential equations to be solved using a stabilized finite-difference scheme. The integral component of the equation is approximated using an HermiteGauss quadrature. ployed in practice as an alternative to pricing derivative securities using finite-difference techniques, particularly in the case of American-style options.

\section{REFERENCES}

[1] Y. Aït-Sahalia and J. Jacod, "Analyzing the Spectrum of Asset Returns: Jump and Volatility Components in High Frequency Data," Journal of Economic Literature, Vol. 50, No. 4, 2012, pp. 1007-1050.

[2] S. L. Heston, "A Closed-Form Solution for Options with Stochastic Volatility with Applications to Bond and Currency Options," Review of Financial Studies, Vol. 6, No. 2, 1993, pp. 327-343. doi:10.1093/rfs/6.2.327

[3] F. Black and M. Scholes, "The Pricing of Options and Corporate Liabilities," Journal of Political Economy, Vol. 81, No. 3, 1973, pp. 637-654. doi:10.1086/260062

[4] D. S. Bates, "Jumps and Stochastic Volatility: Exchange Rate Processes Implicit in Deutsche Mark Options," Review of Financial Studies, Vol. 9, No. 1, 1996, pp. 69-107. doi:10.1093/rfs/9.1.69

[5] Y. Aït-Sahalia and J. Jacod, "Testing for Jumps in a Discretely Observed Process," Annals of Statistics, Vol. 37, No. 1, 2009, pp. 184-222. doi:10.1214/07-AOS568

[6] D. Duffie, J. Pan and K. Singleton, "Transform Analysis and Asset Pricing for Affine Jump-Diffusions," Econometrica, Vol. 68, No. 6, 2000, pp. 1343-1376. doi:10.1111/1468-0262.00164

[7] G. Bakshi and D. Madan, "Spanning and Derivative-Security Valuation," Journal of Financial Economics, Vol. 55, No. 2, 2000, pp. 205-238. doi:10.1016/S0304-405X(99)00050-1

[8] R. Geske and H. E. Johnson, "The American Put Option Valued Analytically," Journal of Finance, Vol. 39, No. 5, 1984, pp. 1511-1524.

[9] C. Chiarella, B. Kang, G. H. Meyer and A. Ziogas, "The Evaluation of American Option Prices under Stochastic Volatility and Jump-Diffusion Dynamics Using the Method of Lines," Quantitative Finance Research Centre, University of Technology, Sydney, 2008.

[10] O. Zhylyevskyy, "A Fast Fourier Transform Technique for Pricing American Options under Stochastic Volatility," Review of Derivatives Research, Vol. 13, No. 1, 2010, pp. 1-24. doi:10.1007/s11147-009-9041-6

[11] J. M. Harrison and D. M. Kreps, "Martingales and Arbitrage in Multiperiod Securities Markets," Journal of Economic Theory, Vol. 20, No. 3, 1979, pp. 381-408. doi:10.1016/0022-0531(79)90043-7

[12] M. Chernov and E. Ghysels, "A Study towards a Unified Approach to the Joint Estimation of Objective and Risk Neutral Measures for the Purpose of Options Valuation," Journal of Financial Economics, Vol. 56, No. 3, 2000, pp. 407-458. doi:10.1016/S0304-405X(00)00046-5

[13] P. Protter, "Stochastic Integration and Differential Equations: A New Approach," Springer-Verlag, New York, 1990.

[14] K. L. Chung, “A Course in Probability Theory,” 3rd Edi- 
tion, Academic Press, San Diego, 2001.

[15] O. Zhylyevskyy, "Efficient Pricing of European-Style Options under Heston's Stochastic Volatility Model," Theoretical Economics Letters, Vol. 2, No. 1, 2012, pp. 16-20. doi:10.4236/tel.2012.21003

[16] R. C. Merton, "Theory of Rational Option Pricing," Bell Journal of Economics and Management Science, Vol. 4, No. 1, 1973, pp. 141-183. doi:10.2307/3003143

[17] W. H. Press, S. A. Teukolsky, W. T. Vetterling and B. P. Flannery, "Numerical Recipes in Fortran 77: The Art of
Scientific Computing," 2nd Edition, Cambridge University Press, Cambridge, 2001.

[18] N. G. Shephard, "From Characteristic Function to Distribution Function: A Simple Framework for the Theory," Econometric Theory, Vol. 7, No. 4, 1991, pp. 519-529. doi:10.1017/S0266466600004746

[19] T. W. Epps, "Pricing Derivative Securities," World Scientific, River Edge, 2000. 